\title{
Methods for Estimating Specific Loss Power in Magnetic Hyperthermia Revisited
}

\section{Kenya Murase}

Department of Medical Physics and Engineering, Division of Medical Technology and Science, Faculty of Health Science, Graduate School of Medicine, Osaka University, Osaka, Japan

Email: murase@sahs.med.osaka-u.ac.jp

How to cite this paper: Murase, K. (2016) Methods for Estimating Specific Loss Power in Magnetic Hyperthermia Revisited. Open Journal of Applied Sciences, 6, 815-825. http://dx.doi.org/10.4236/ojapps.2016.612071

Received: October 10, 2016

Accepted: November 8, 2016

Published: November 11, 2016

Copyright $\odot 2016$ by author and Scientific Research Publishing Inc. This work is licensed under the Creative Commons Attribution International License (CC BY 4.0).

http://creativecommons.org/licenses/by/4.0/

\section{Abstract}

Our purpose in this study was to present three methods for estimating specific loss power (SLP) in magnetic hyperthermia with use of an alternating magnetic field (AMF) and magnetic nanoparticles (MNPs) and to compare the SLP values estimated by the three methods using simulation studies under various diameters of MNPs $(D)$, amplitudes $\left(H_{0}\right)$ and frequencies of AMF $(f)$. In the first method, the SLP was calculated by solving the magnetization relaxation equation of Shliomis numerically $\left(S L P_{1}\right)$. In the second method, the SLP was obtained by solving Shliomis' relaxation equation using the complex susceptibility $\left(S L P_{2}\right)$. The third method was based on Rosensweig's model $\left(S L P_{3}\right)$. The $S L P_{3}$ value changed largely depending on the magnetic field strength $(H)$ in the Langevin parameter $(\xi)$ and it became maximum $\left(S L P_{3}^{\max }\right)$ and minimum $\left(S L P_{3}^{\min }\right)$ when $H$ was 0 and $\pm H_{0}$, respectively. The relative difference between $S L P_{1}$ and $S L P_{2}$ was the largest and increased with increasing $D$ and $H_{0}$, whereas that between $S L P_{1}$ and $S L P_{3}^{\min }$ was the smallest and was almost constant regardless of $D$ and $H_{0}$, suggesting that $H$ in $\xi$ should be taken as $H_{0}$ in estimating the SLP using Rosensweig's model. In conclusion, this study will be useful for optimizing the parameters of AMF in magnetic hyperthermia and for the optimal design of MNPs for magnetic hyperthermia.

\section{Keywords}

Magnetic Hyperthermia, Magnetic Nanoparticle, Specific Loss Power, Alternating Magnetic Field, Magnetization Relaxation

\section{Introduction}

Hyperthermia is one of the promising approaches to cancer therapy. The most com- 
monly used heating method in the clinical setting is capacitive heating that uses a radiofrequency (RF) electric field [1]. However, a major technical problem with hyperthermia is the difficulty of heating the targeted tumor to the desired temperature without damaging the surrounding tissues, as the electromagnetic energy must be directed from an external source and penetrate normal tissue. Other hyperthermia modalities, including ultrasound hyperthermia, have been reported [2], but the efficacy of these modalities depends on the size and depth of the tumor, and disadvantages include the ability to target the tumor and control the exposure.

Hyperthermia with use of magnetic nanoparticles (MNPs) (magnetic hyperthermia) was developed in the 1950s [3] and is still under development in the effort to overcome the above disadvantages [4] [5]. MNPs generate heat in an alternating magnetic field (AMF) as a result of hysteresis and relaxational losses, which results in heating of the tissue in which MNPs accumulate [6]. For small MNPs, the relaxational losses caused by a delay in magnetization relaxation are dominant for heat dissipation [6]. With the development of precise methods for synthesizing functionalized MNPs [7], MNPs with functionalized surfaces, which have high specificity for tumor tissue, have been developed as heating elements for magnetic hyperthermia [8]. Recently, MNPs with a higher heating efficiency, i.e., specific loss power (SLP), have also been actively developed [9]. Furthermore, there is renewed interest in magnetic hyperthermia as a treatment modality for cancer, especially when it is combined with other, more traditional therapeutic approaches such as the co-delivery of anticancer drugs [10] or radiation therapy [11]. From these aspects, magnetic hyperthermia has received much recent attention.

The estimation of SLP is important for evaluating the heating efficiency of MNPs, for optimizing the parameters of AMF, and for the optimal design of MNPs in an attempt to establish the effectiveness of magnetic hyperthermia. Rosensweig's model [6] has often been used for the estimation of SLP. His model, however, is based on the so-called linear magnetization assumption [12], and thus it is said that his model is strictly valid only in the limit of small amplitude and frequency of AMF. In this study, we presented three methods for estimating SLP and compared the SLP values estimated by the three methods under various conditions of MNPs and AMF. Especially, we investigated the validity of Rosensweig's model in comparison with the numerical solution of the magnetization relaxation equation of Shliomis [13].

\section{Materials and Methods}

\subsection{Theory}

The magnetization relaxation equation of Shliomis [12] [13] is given by

$$
\frac{\mathrm{d} \boldsymbol{M}}{\mathrm{d} t}=\boldsymbol{\Omega} \times \boldsymbol{M}-\frac{\boldsymbol{M}-\boldsymbol{M}_{0}}{\tau}-\frac{\boldsymbol{M} \times(\boldsymbol{M} \times \boldsymbol{H})}{6 \eta \phi},
$$

where $M$ is the magnetization of MNPs under the magnetic field $H, \Omega$ is the flow velocity, $\phi$ is the volume fraction, and $\eta$ is the viscosity of the suspending fluid. When there is no bulk flow and $\boldsymbol{M}$ and $H$ are collinear, Equation (1) is reduced to the following eq- 
uation [6]:

$$
\frac{\mathrm{d} M(t)}{\mathrm{d} t}=-\frac{M(t)-M_{0}(t)}{\tau}
$$

In Equation (2), $\tau$ is the effective relaxation time given by

$$
\frac{1}{\tau}=\frac{1}{\tau_{N}}+\frac{1}{\tau_{B}}
$$

where $\tau_{N}$ and $\tau_{B}$ are the Néel relaxation and Brownian relaxation time, respectively [6]. $\tau_{N}$ and $\tau_{B}$ are given by the following relationships [6]:

$$
\tau_{N}=\tau_{0} \frac{\sqrt{\pi} e^{\Gamma}}{2 \sqrt{\Gamma}} \text { and } \tau_{B}=\frac{3 \eta V_{H}}{k_{B} T}
$$

where $\tau_{0}$ is the average relaxation time in response to a thermal fluctuation, $k_{B}$ is the Boltzmann constant, $T$ is the temperature, and $\Gamma=K V_{M} /\left(k_{B} T\right)$, with $K$ being the anisotropy constant of MNP. $V_{H}$ is taken as the hydrodynamic volume of MNP that is larger than the magnetic volume $V_{M}=\pi D^{3} / 6$ for MNP of diameter $D$. As a model for $V_{H}$, it is assumed that $V_{H}=(1+2 \delta / D)^{3} V_{M}$, where $\delta$ is the thickness of a sorbed surfactant layer [6]. $M_{0}(t)$ in Equation (2) denotes the equilibrium magnetization and is given by

$$
M_{0}(t)=\chi_{0} H(t)
$$

where $\chi_{0}$ is the equilibrium susceptibility. In this study, $H(t)$ was assumed to be

$$
H(t)=H_{0} \cos (2 \pi f t)
$$

where $H_{0}$ and $f$ denote the amplitude and frequency of AMF, respectively. Because the actual equilibrium susceptibility $\left(\chi_{0}\right)$ is dependent on the magnetic field, $\chi_{0}$ was assumed to be the chord susceptibility corresponding to the Langevin equation, given by [6]

$$
\chi_{0}=\chi_{i} \frac{3}{\xi}\left(\operatorname{coth} \xi-\frac{1}{\xi}\right)
$$

where $\chi_{i}$ is the initial susceptibility given by $\chi_{i}=\mu_{0} \phi M_{d}^{2} V_{M} /\left(3 k_{B} T\right), \xi$ is the Langevin parameter given by $\xi=\mu_{0} M_{d} H V_{M} /\left(k_{B} T\right), M_{d}$ is the domain magnetization of a suspended particle, and $\mu_{0}$ is the permeability of free space. It should be noted that $\xi$ is magnetic field $(H)$ dependent and thus time dependent.

Solving Equation (2) and using Equation (5) and Equation (6) yield

$$
M(t)=\frac{1}{\tau} \mathrm{e}^{-\frac{t}{\tau}} \otimes M_{0}(t)+M(0) \mathrm{e}^{-\frac{t}{\tau}}=\frac{1}{\tau} \mathrm{e}^{-\frac{t}{\tau}} \otimes \chi_{0} H_{0} \cos (2 \pi f t)+M(0) \mathrm{e}^{-\frac{t}{\tau}},
$$

where $\otimes$ denotes the convolution integral and $M(0)$ is $M(t)$ at $t=0$. In this study, $M(0)$ was assumed to be $M(0)=M_{0}(0)=\chi_{0} H_{0}$. When $t=\infty$, however, the second term of the right-hand side of Equation (8) can be neglected. It should be noted that if we calculate $M(t)$ as a function of $H(t)$, we can obtain the hysteresis loop, i.e., M-H curve.

According to Rosensweig [6], solving Equation (2) using the complex susceptibility 
given by $\chi=\chi^{\prime}-j \chi^{\prime \prime}(j=\sqrt{-1})$ and Equation (5) and Equation (6) with an assumption that $\chi_{0}$ is constant, yields

$$
M(t)=H_{0}\left[\chi^{\prime} \cos (2 \pi f t)+\chi^{\prime \prime} \sin (2 \pi f t)\right],
$$

where $\chi^{\prime}$ (in-phase component) and $\chi^{\prime \prime}$ (out-of-phase component) are, respectively, given by [6]

$$
\chi^{\prime}=\frac{\chi_{0}}{1+(2 \pi f \tau)^{2}} \text { and } \chi^{\prime \prime}=\frac{2 \pi f \tau \chi_{0}}{1+(2 \pi f \tau)^{2}} .
$$

The average rate of energy dissipation per cycle of the period, i.e., $1 / f(\langle P\rangle)$ is given by [6]

$$
\langle P\rangle=-\mu_{0} f \int_{0}^{1 / f} M(t) \frac{\mathrm{d} H(t)}{\mathrm{d} t} \mathrm{~d} t .
$$

Substituting Equation (6) into Equation (11) yields

$$
\langle P\rangle=2 \pi \mu_{0} H_{0} f^{2} \int_{0}^{1 / f} M(t) \sin (2 \pi f t) \mathrm{d} t .
$$

The rate of energy dissipation per unit mass of MNPs, i.e., specific loss power (SLP) can be obtained from $\langle P\rangle$ as [12]

$$
S L P=\frac{\langle P\rangle}{\phi \rho},
$$

where $\rho$ is the density of suspending fluid.

In this study, we considered the following three methods for estimating SLP. In the first method, Equation (8) was used for $M(t)$ in Equation (12). In this case, because $M(t)$ must be time-periodic in the steady state, the SLP value for the $i$-th cycle of the $\mathrm{M}-\mathrm{H}$ curve (denoted by $S L P_{1}^{i}$ ) can be given by

$$
S L P_{1}^{i}=\frac{2 \pi \mu_{0} H_{0} f^{2}}{\phi \rho}\left\{\int_{(i-1) / f}^{i / f}\left[\frac{1}{\tau} \mathrm{e}^{-\frac{t}{\tau}} \otimes M_{0}(t)\right] \sin (2 \pi f t) \mathrm{d} t+\frac{2 \pi f \tau^{2} M(0)}{1+(2 \pi f \tau)^{2}} \mathrm{e}^{-\frac{i}{f \tau}}\left(\mathrm{e}^{\frac{1}{f \tau}}-1\right)\right\}
$$

It should be noted that when $i$ is sufficiently large, the second term of the right-hand side of Equation (14) can be neglected and $S L P_{1}^{i}$ approaches the steady state. We denote the $S L P_{1}^{i}$ value in the quasi steady state by $S L P_{1}$. Actually, $S L P_{1}$ was taken as the $S L P_{1}^{i}$ value in the case when $\left|S L P_{1}^{i}-S L P_{1}^{i-1}\right| / S L P_{1}^{i-1}<\varepsilon$, with $\varepsilon$ being taken as $10^{-6}$, where $|*|$ denotes the absolute value. The integration in Equation (14) was performed by use of the trapezoidal rule [14] ("trapz" in MATLAB'; The MathWorks, Inc., Natick, MA, USA) and the convolution integral was calculated using the MATLAB ${ }^{\circledR}$ function ("conv").

In the second method, Equation (9) was used for $M(t)$ in Equation (12). In this case, the SLP value (denoted by $S L P_{2}$ ) can be given by

$$
S L P_{2}=\frac{2 \pi \mu_{0} H_{0}^{2} f^{2}}{\phi \rho} \int_{0}^{1 / f}\left[\chi^{\prime} \cos (2 \pi f t)+\chi^{\prime \prime} \sin (2 \pi f t)\right] \sin (2 \pi f t) \mathrm{d} t .
$$

As in Equation (14), the integration in Equation (15) was also performed by use of the 
trapezoidal rule [14] (“trapz" in MATLAB ${ }^{\otimes}$; The MathWorks, Inc., Natick, MA, USA).

In the third method, $\chi^{\prime}$ and $\chi^{\prime \prime}$ (basically $\chi_{0}$ ) were assumed to be constant in Equation (15), though they are actually magnetic field $(H)$ dependent. In this case, the SLP value (denoted by $S L P_{3}$ ) can be obtained from Equation (10) and Equation (15) as

$$
S L P_{3}=\frac{2 \pi \mu_{0} H_{0}^{2} f^{2}}{\phi \rho} \chi^{\prime \prime} \int_{0}^{1 / f} \sin (2 \pi f t)^{2} \mathrm{~d} t=\frac{\pi \mu_{0} \chi_{0} H_{0}^{2} f}{\phi \rho} \frac{2 \pi f \tau}{1+(2 \pi f \tau)^{2}} .
$$

It should be noted that $S L P_{3} \times \phi \rho$ is equal to the equation for the energy dissipation derived by Rosensweig [6]. As shown afterwards, $S L P_{3}$ changes depending on the magnetic field strength. Thus, we denote the maximum, minimum, and mean $S L P_{3}$ values in a cycle of the period, i.e., $1 / f$ by $S L P_{3}^{\max }, S L P_{3}^{\min }$, and $S L P_{3}^{\operatorname{mean}}$, respectively. $S L P_{3}^{\text {mean }}$ was calculated from

$$
S L P_{3}^{\text {mean }}=f \int_{0}^{1 / f} S L P_{3} \mathrm{~d} t
$$

For comparison of $S L P_{1}, S L P_{2}, S L P_{3}^{\max }, S L P_{3}^{\min }$, and $S L P_{3}^{\text {mean }}$, we calculated the relative differences $\left(R D_{2}, R D_{3}^{\max }, R D_{3}^{\min }\right.$, and $\left.R D_{3}^{\text {mean }}\right)$ defined by

$$
\begin{aligned}
R D_{2} & =\frac{S L P_{2}-S L P_{1}}{S L P_{1}}, \\
R D_{3}^{\max } & =\frac{S L P_{3}^{\max }-S L P_{1}}{S L P_{1}}, \\
R D_{3}^{\min } & =\frac{S L P_{3}^{\min }-S L P_{1}}{S L P_{1}},
\end{aligned}
$$

and

$$
R D_{3}^{\text {mean }}=\frac{S L P_{3}^{\text {mean }}-S L P_{1}}{S L P_{1}}
$$

\subsection{Simulation Studies}

In this study, we assumed that MNPs consisted of maghemite $\left(\gamma-\mathrm{Fe}_{2} \mathrm{O}_{3}\right)$ and fixed $\tau_{0}, \delta$, $M_{d}, K, \eta, \rho, \phi$, and $T$ to be $10^{-9} \mathrm{~s}, 2 \mathrm{~nm}, 414 \mathrm{kA} / \mathrm{m}, 4.7 \mathrm{~kJ} / \mathrm{m}^{3}, 0.00235 \mathrm{~kg} / \mathrm{m} / \mathrm{s}, 4600$ $\mathrm{kg} / \mathrm{m}^{3}, 0.003$, and $37^{\circ} \mathrm{C}$, respectively [15]. When $H_{0}, f$, and $D$ were fixed, they were taken as $20 \mathrm{mT}, 300 \mathrm{kHz}$, and $20 \mathrm{~nm}$, respectively. It should be noted that the unit of $\mathrm{mT}$ can be converted to $\mathrm{kA} / \mathrm{m}$ by use of the relationship $1 \mathrm{mT}=0.796 \mathrm{kA} / \mathrm{m}$.

\section{Results}

As shown in Equation (14), the $S L P_{1}^{i}$ value depends on the cycle number of the M-H curve. Thus, we calculated the $S L P_{1}^{i}$ value in the quasi steady state, i.e., the $S L P_{1}$ value under the condition of $\left|S L P_{1}^{i}-S L P_{1}^{i-1}\right| / S L P_{1}^{i-1}<\varepsilon$ with $\varepsilon$ being taken as $10^{-6}$, as previously described. When we neglected the second term in the right-hand side of Equation (14), the $S L P_{1}^{i}$ value reached the steady state after a few cycles in all the cases studied. Figure 1 (a) shows the M-H curves in the quasi steady state calculated from Equa- 
tion (8) for various frequencies of AMF. For comparison, Figure 1(b) shows the M-H curves calculated from Equation (9). It should be noted that $M(t)$ was normalized by the saturation magnetization $\left(M_{s}\right)$ given by $M_{s}=\phi M_{d}$. In these simulations, $H_{0}$ was fixed at $20 \mathrm{mT}$ and $D$ was assumed to be $20 \mathrm{~nm}$. Figure 2 shows the case when $D$ was varied from $10 \mathrm{~nm}$ to $30 \mathrm{~nm}$ with steps of $5 \mathrm{~nm}$. As shown in Figure 1 and Figure 2, a large difference between the M-H curves obtained by Equation (8) and Equation (9) was observed and it increased with increasing $f$ and $D$.

Figure 3(a) shows the $S L P_{3}$ values calculated from Equation (16) as a function of $H$ with $f$ being varied from $200 \mathrm{kHz}$ to $1000 \mathrm{kHz}$ with steps of $200 \mathrm{kHz}$, whereas Figure 3(b) shows the case when $D$ was varied from $10 \mathrm{~nm}$ to $30 \mathrm{~nm}$ with steps of $5 \mathrm{~nm}$. In

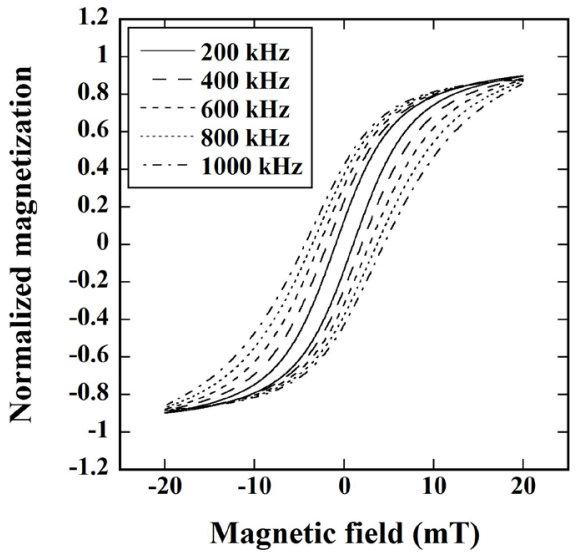

(a)

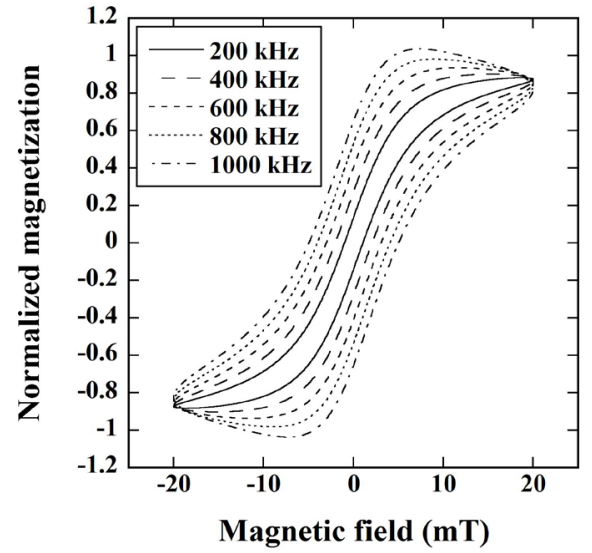

(b)

Figure 1. (a) M-H curves (hysteresis loops) in the quasi steady state calculated from Equation (8) for various frequencies of an alternating magnetic field (AMF) ( $f$ ); (b) M-H curves calculated from Equation (9) for various $f$. In these simulations, the amplitude of AMF $\left(H_{0}\right)$ and diameter of magnetic nanoparticles $(D)$ were assumed to be $20 \mathrm{mT}$ and $20 \mathrm{~nm}$, respectively. Note that the unit of $\mathrm{mT}$ can be converted to $\mathrm{kA} / \mathrm{m}$ by use of the relationship $1 \mathrm{mT}=0.796 \mathrm{kA} / \mathrm{m}$.

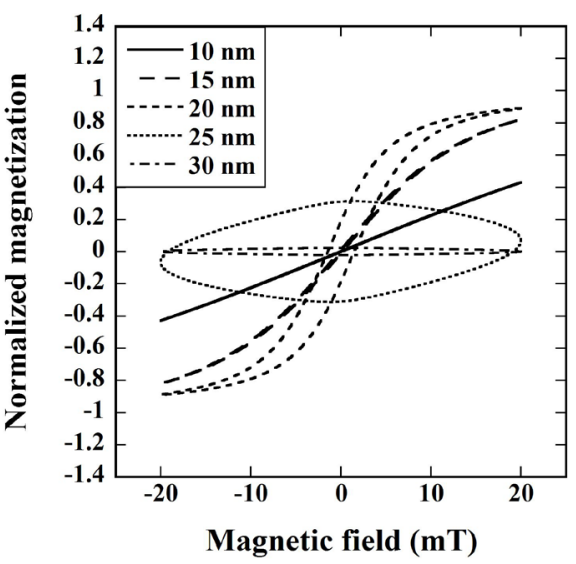

(a)

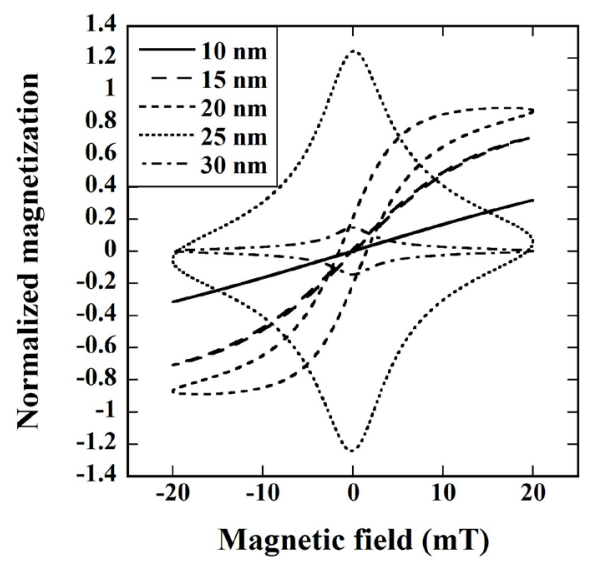

(b)

Figure 2. (a) M-H curves in the quasi steady state calculated from Equation (8) for various $D$; (b) M-H curves calculated from Equation (9) for various $D$. In these simulations, $H_{0}$ and $f$ were assumed to be $20 \mathrm{mT}$ and $300 \mathrm{kHz}$, respectively. 
these simulations, $H_{0}$ was fixed at $20 \mathrm{mT}$. As shown in Figure 3, the $S L P_{3}$ value became maximum, i.e., $S L P_{3}^{\max }$ when $H$ was zero. When $|H|$ was the maximum, i.e., $H_{0}$, the $S L P_{3}$ value became minimum, i.e., $S L P_{3}^{\min }$.

Figure 4(a) shows the comparison of $S L P_{1}, S L P_{2}, S L P_{3}^{\max }, S L P_{3}^{\min }$, and $S L P_{3}^{\text {mean }}$ as a function of $D$, whereas Figure 4 (b) shows the $R D_{2}, R D_{3}^{\max }, R D_{3}^{\min }$, and $R D_{3}^{\text {mean }}$ values as a function of $D$. As shown in Figure 4(b), $R D_{3}^{\max }$ was the largest and

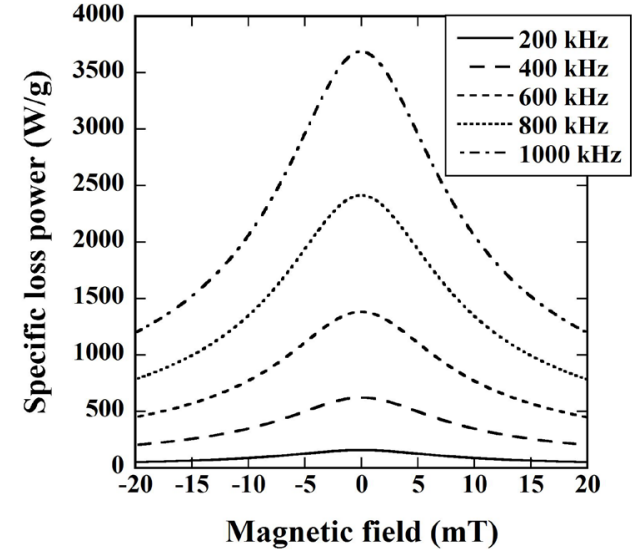

(a)

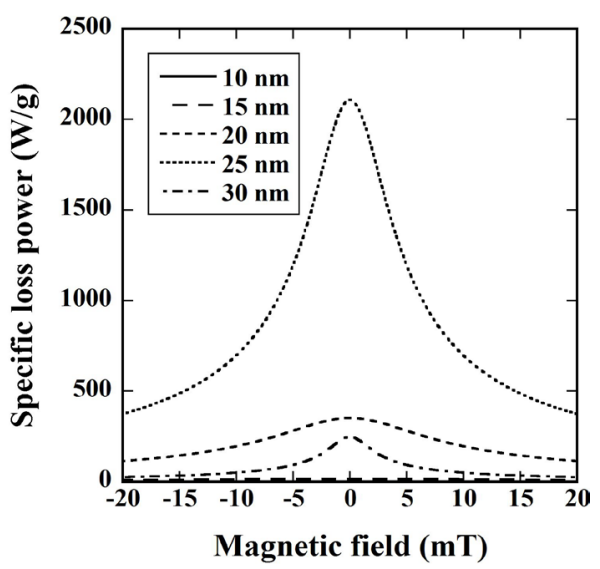

(b)

Figure 3. (a) Specific loss power (SLP) values calculated from Equation (16) $\left(S L P_{3}\right)$ as a function of the magnetic field $(H)$ for various $f$. In these simulations, $H_{0}$ and $D$ were assumed to be $20 \mathrm{mT}$ and $20 \mathrm{~nm}$, respectively; (b) $S L P_{3}$ values calculated from Equation (16) as a function of $H$ for various $D$. In these simulations, $H_{0}$ and $f$ were assumed to be $20 \mathrm{mT}$ and $300 \mathrm{kHz}$, respectively. Note that the $S L P_{3}$ values for $D$ of $10 \mathrm{~nm}$ and $15 \mathrm{~nm}$ are too small to be seen in the figure.

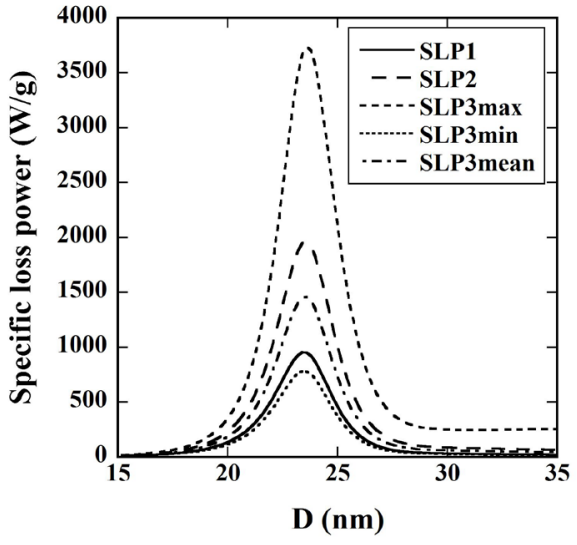

(a)

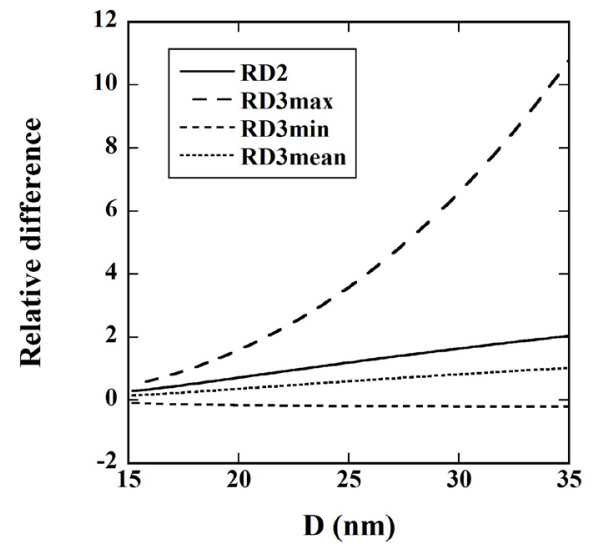

(b)

Figure 4. (a) $S L P_{1}, S L P_{2}, S L P_{3}^{\max }, S L P_{3}^{\min }$, and $S L P_{3}^{\text {mean }}$ values as a function of $D$. Note that $S L P_{1}$ and $S L P_{2}$ were calculated from Equation (14) and Equation (15), respectively. $S L P_{3}^{\max }$ and $S L P_{3}^{\min }$ denote the maximum and minimum values of $S L P_{3}$ calculated from Equation (16), respectively. $S L P_{3}^{\text {mean }}$ was calculated from Equation (17); (b) $R D_{2}, R D_{3}^{\max }, R D_{3}^{\min }$, and $R D_{3}^{\text {mean }}$ values as a function of $D$. Note that $R D_{2}, R D_{3}^{\max }, R D_{3}^{\min }$, and $R D_{3}^{\text {mean }}$ represent the relative differences calculated from Equation (18), Equation (19), Equation (20), and Equation (21), respectively. In these simulations, $H_{0}$ and $f$ were assumed to $20 \mathrm{mT}$ and $300 \mathrm{kHz}$, respectively. 
increased with increasing $D$, whereas $R D_{3}^{\min }$ was the smallest and was almost constant regardless of $D$.

Figure 5(a) shows the comparison of $S L P_{1}, S L P_{2}, S L P_{3}^{\max }, S L P_{3}^{\min }$, and $S L P_{3}^{\text {mean }}$ as a function of $H_{0}$, whereas Figure 5 (b) shows the $R D_{2}, R D_{3}^{\max }, R D_{3}^{\min }$, and $R D_{3}^{\text {mean }}$ values as a function of $H_{0}$. As shown in Figure 5(b), $R D_{3}^{\max }$ was the largest and increased with increasing $H_{0}$, whereas $R D_{3}^{\min }$ was the smallest and was almost constant regardless of $H_{0}$.

Figure 6(a) shows the comparison of $S L P_{1}, S L P_{2}, S L P_{3}^{\max }, S L P_{3}^{\min }$, and $S L P_{3}^{\text {mean }}$ as a function of $f$, whereas Figure $6(\mathrm{~b})$ shows the $R D_{2}, R D_{3}^{\max }, R D_{3}^{\min }$, and $R D_{3}^{\text {mean }}$ values as a function of $f$. In this case, the $R D_{2}, R D_{3}^{\max }, R D_{3}^{\min }$, and $R D_{3}^{\text {mean }}$ values were almost constant regardless of $f$ (Figure 6(b)).

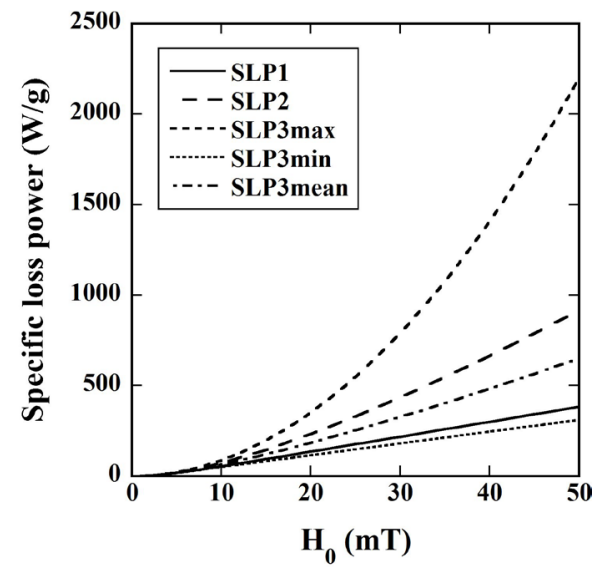

(a)

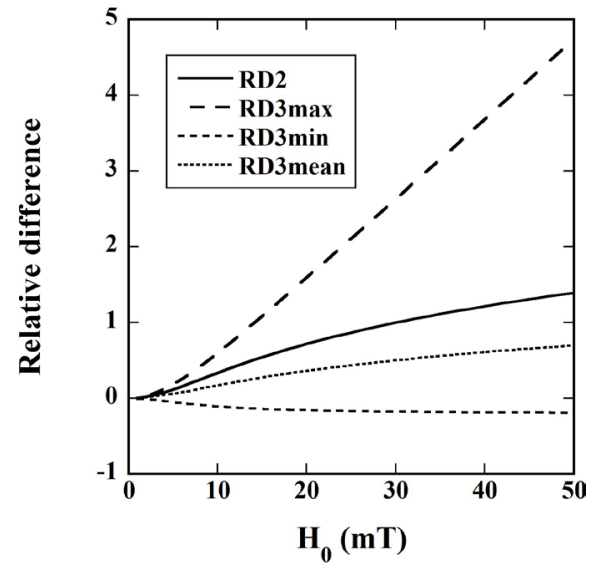

(b)

Figure 5. (a) $S L P_{1}, S L P_{2}, S L P_{3}^{\max }, S L P_{3}^{\min }$, and $S L P_{3}^{\text {mean }}$ values as a function of $H_{0}$; (b) $R D_{2}$, $R D_{3}^{\max }, R D_{3}^{\min }$, and $R D_{3}^{\text {mean }}$ values as a function of $H_{0}$. In these simulations, $D$ and $f$ were assumed to be $20 \mathrm{~nm}$ and $300 \mathrm{kHz}$, respectively.

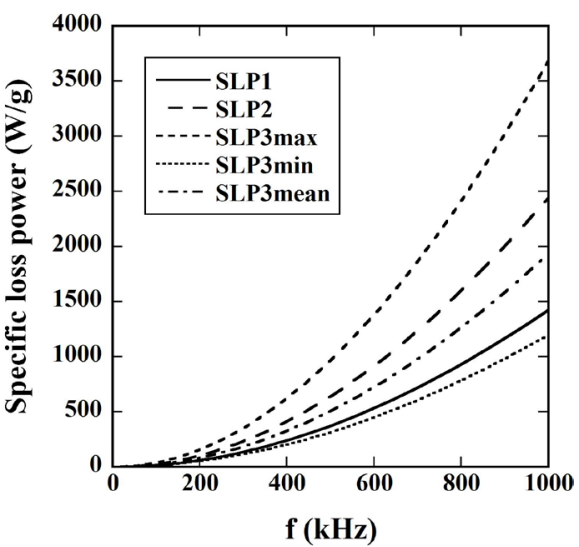

(a)

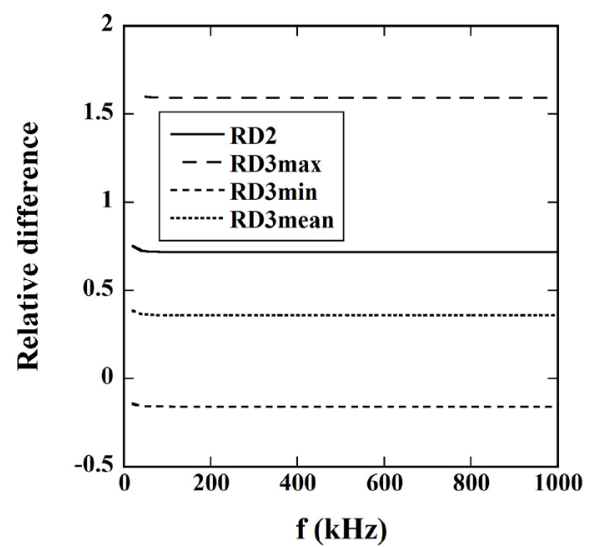

(b)

Figure 6. (a) $S L P_{1}, S L P_{2}, S L P_{3}^{\max }, S L P_{3}^{\min }$, and $S L P_{3}^{\text {mean }}$ values as a function of $f ;$ (b) $R D_{2}$, $R D_{3}^{\max }, R D_{3}^{\min }$, and $R D_{3}^{\operatorname{mean}}$ values as a function of $f$. In these simulations, $H_{0}$ and $D$ were assumed to be $20 \mathrm{mT}$ and $20 \mathrm{~nm}$, respectively. 


\section{Discussion}

In this study, we presented three methods for the estimation of SLP in magnetic hyperthermia and compared the SLP values estimated by the three methods $\left(S L P_{1}, S L P_{2}\right.$, and $\left.S L P_{3}\right) . S L P_{1}$ was derived by solving the magnetization relaxation equation of Shliomis [13] numerically. $S L P_{2}$ was derived by solving Shliomis' relaxation equation [13] using the complex susceptibility. $S L P_{3}$ was derived based on Rosensweig's model, in which the complex susceptibility with $\chi^{\prime}$ and $\chi^{\prime \prime}$ (basically $\chi_{0}$ ) being assumed to be constant has been used.

As previously described, Rosensweig's model [6] has often been used for the estimation of SLP. To the best of our knowledge, however, few studies have been performed to validate the SLP estimation based on Rosensweig's method [6] in comparison with that based on the numerical solution of the magnetization relaxation equation of Shliomis [13].

As shown in Figure 1 and Figure 2, a large difference was observed between the $\mathrm{M}-\mathrm{H}$ curves calculated from Equation (8) and Equation (9), especially when $H$ is zero, and the difference increased with increasing $f$ and $D$. When using Equation (9), $M(t)$ becomes equal to $\chi^{\prime} H_{0}$ at $t=0$, i.e., $H=H_{0}$ and it becomes equal to $-\chi^{\prime} H_{0}$ at $t=1 /(2 f)$, i.e., $H=-H_{0}$. On the other hand, $M(t)$ becomes equal to $\chi^{\prime \prime} H_{0}$ at $t=1 /(4 f)$, i.e., $H=0$ and it becomes equal to $-\chi^{\prime \prime} H_{0}$ at $t=3 /(4 f)$, i.e., $H=0$. Thus, the above difference in the $\mathrm{M}-\mathrm{H}$ curves shown in Figure 1 and Figure 2 may suggest that $\chi^{\prime \prime}$ given by Equation (10) is overestimated compared to the case when using Equation (8). Furthermore, the area of the M-H curve calculated from Equation (9) (Figure 1(b) and Figure 2(b)) was larger than that calculated from Equation (8) (Figure 1(a) and Figure 2(a)). The area of the M-H curve directly represents the power loss during one cycle of the hysteresis loop. Thus, the above finding corresponds to the fact that $S L P_{2}$ is larger than $S L P_{1}$ (Figure 4(a), Figure 5(a), and Figure 6(a)).

The $S L P_{3}$ given by Equation (16) has often been used for characterizing the heating property of MNPs [16]. As previously described, $S L P_{3}$ has been derived with an assumption that $\chi_{0}$ is constant. However, $\chi_{0}$ is actually magnetic field $(H)$ dependent, because $\chi_{0}$ is the function of the Langevin parameter $(\xi)$ as shown in Equation (7) and $\xi$ is the function of $H$. To investigate to what extent $S L P_{3}$ depends on $H$, we showed the $S L P_{3}$ values as a function of $H$ in Figure 3. As shown in Figure 3, the $S L P_{3}$ value changed largely depending on $H . \chi_{0}$ in Equation (16) is the monotonically decreasing function of $|\xi|$ or $|H|$ (data not shown). Thus, the $S L P_{3}$ value becomes maximum when $H$ is zero (Figure 3 ). In this case, $\chi_{0}$ becomes equal to $\chi_{p}$ because coth $\xi-1 / \xi$ in Equation (7) approaches $\xi / 3$ when $\xi$ approaches zero. On the other hand, when $|H|$ is maximum, i.e., $H$ is equal to $\pm H_{0}$, the $S L P_{3}$ value becomes minimum.

To compare the SLP values estimated by Equation (15) and Equation (16) with that estimated using the numerical solution of the magnetization relaxation equation of Shliomis [13], we calculated the relative differences given by Equation (18) to Equation (21). As shown in Figure 4 and Figure 5 , the $R D_{3}^{\max }$ value was the largest and increased with increasing $D$ and $H_{0}$, whereas the $R D_{3}^{\min }$ value was the smallest and was 
almost constant regardless of $D$ and $H_{0}$. These results suggest that when estimating SLP using Rosensweig's model [6], $H$ in $\xi$ should be taken as $H_{0}$.

In this study, we solved the magnetization relaxation equation of Shliomis [13] (Equation (1)) with an assumption that there is no bulk flow and the magnetization of MNPs and magnetic field are collinear. In this case, Equation (1) is reduced to Equation (2), which can be easily solved using convolution integral as shown in Equation (8). Although Equation (2) appears to be valid in considering the magnetic hyperthermia with use of small MNPs in the superparamagnetic state and we believe that this study will provide the basis for establishing the effectiveness of such magnetic hyperthermia, it will be necessary to solve Equation (1) without any assumptions or another magnetization equation derived microscopically from the Fokker-Planck equation [12] [17] for more detailed analysis. These studies are currently in progress. As previously described, we targeted the MNPs consisting of maghemite with the magnetic and physical properties described in the "Simulation Studies" section, because maghemite is the core iron oxide of Resovist ${ }^{\oplus}$, which is a commercially-available organ-specific contrast agent for magnetic resonance imaging and has been approved for clinical use in Japan [15]. We will also perform further studies for other MNPs.

\section{Conclusion}

We presented three methods for estimating SLP in magnetic hyperthermia and compared the SLP values estimated by the three methods under various conditions of MNPs and AMF. This study will be useful for optimizing the parameters of AMF in magnetic hyperthermia and for developing the MNPs suitable for magnetic hyperthermia. We also investigated the validity of Rosensweig's model in comparison with the numerical solution of the magnetization relaxation equation of Shliomis, suggesting that when estimating SLP using Rosensweig's model, the magnetic field strength in the Langevin parameter should be taken as the amplitude of AMF.

\section{Acknowledgements}

This work was supported by a Grant-in-Aid for Scientific Research (Grant Number: 25282131 and 15K12508) from the Japan Society for the Promotion of Science (JSPS).

\section{References}

[1] Abe, M., Hiraoka, M., Takahashi, M., Egawa, S., Matsuda, C., Onoyama, Y., Morita, K., Kakehi, M. and Sugahara, T. (1986) Multi-Institutional Studies on Hyperthermia Using an 8$\mathrm{MHz}$ Radiofrequency Capacitive Heating Device (Thermotron RF-8) in Combination with Radiation for Cancer Therapy. Cancer, 58, 1589-1595. http://dx.doi.org/10.1002/1097-0142(19861015)58:8<1589::AID-CNCR2820580802>3.0.CO; $\underline{2-\mathrm{B}}$

[2] Seip, R. and Ebbini, E.S. (1995) Noninvasive Estimation of Tissue Temperature Response to Heating Fields Using Diagnostic Ultrasound. IEEE Transactions on Biomedical Engineering, 42, 828-839. http://dx.doi.org/10.1109/10.398644

[3] Gilchrist, R.K., Medal, R., Shorey, W.D., Hanselman, R.C., Parrott, J.C. and Taylor, C.B. 
(1957) Selective Inductive Heating of Lymph Nodes. Annals of Surgery, 146, 596-606. http://dx.doi.org/10.1097/00000658-195710000-00007

[4] Jordan, A., Scholz, R., Maier-Hauff, K., Johannsen, M., Wust, P., Nodobny, J., Schirra, H., Schmidt, H., Deger, S., Loening, S., Lanksch, W. and Felix, R. (2001) Presentation of a New Magnetic Field Therapy System for the Treatment of Human Solid Tumors with Magnetic Fluid Hyperthermia. Journal of Magnetism and Magnetic Materials, 225, 118-126. http://dx.doi.org/10.1016/S0304-8853(00)01239-7

[5] Murase, K., Aoki, M., Banura, N., Nishimoto, K., Mimura, A., Kuboyabu, T. and Yabata, I. (2015) Usefulness of Magnetic Particle Imaging for Predicting the Therapeutic Effect of Magnetic Hyperthermia. Open Journal of Medical Imaging, 5, 85-99. http://dx.doi.org/10.4236/ojmi.2015.52013

[6] Rosensweig, R.E. (2002) Heating Magnetic Fluid with Alternating Magnetic Field. Journal of Magnetism and Magnetic Materials, 252, 370-374. http://dx.doi.org/10.1016/S0304-8853(02)00706-0

[7] Neuberger, T., Schopf, B., Hofmann, H., Hofmann, M. and von Rechenberga, B. (2005) Superparamagnetic Nanoparticles for Biomedical Applications: Possibilities and Limitations of a New Drug Delivery System. Journal of Magnetism and Magnetic Materials, 293, 483496. http://dx.doi.org/10.1016/j.jmmm.2005.01.064

[8] Ito, A., Shinkai, M., Honda, H. and Kobayashi, T. (2005) Medical Applications of Functionalized Magnetic Nanoparticles. Journal of Bioscience and Bioengineering, 100, 1-11. http://dx.doi.org/10.1263/jbb.100.1

[9] Lee, J.H., Jang, J.T., Choi, J.S., Moon, S.H., Noh, S.H., Kim, J.W., Kim, I.S., Park, K.I. and Cheon, J. (2011) Exchange-Coupled Magnetic Nanoparticles for Efficient Heat Induction. Nature Nanotechnology, 6, 418-422. http://dx.doi.org/10.1038/nnano.2011.95

[10] Kuboyabu, T., Yamawaki, M., Aoki, M., Ohki, A. and Murase, K. (2016) Quantitative Evaluation of Tumor Early Response to Magnetic Hyperthermia Combined with Vascular Disrupting Therapy Using Magnetic Particle Imaging. International Journal of Nanomedicine and Nanosurgery, 2, 1-7. http://dx.doi.org/10.16966/2470-3206.114

[11] Ohki, A., Kuboyabu, T., Aoki, M., Yamawaki, M. and Murase, K. (2016) Quantitative Evaluation of Tumor Response to Combination of Magnetic Hyperthermia Treatment and Radiation Therapy Using Magnetic Particle Imaging. International Journal of Nanomedicine and Nanosurgery, 2, 1-6. http://dx.doi.org/10.16966/2470-3206.117

[12] Soto-Aquino, D. and Rinaldi, C. (2010) Magnetoviscosity in Dilute Ferrofluids from Rotational Brownian Dynamics Simulations. Physical Review E, 82, Article ID: 046310. http://dx.doi.org/10.1103/PhysRevE.82.046310

[13] Shliomis, M.I. (1972) Effective Viscosity of Magnetic Suspensions. Soviet Physics JETP, 34, 1291-1294.

[14] Press, W.H., Teukolsky, S.A., Vetterling, W.T. and Flannery, B.P. (1992) Numerical Recipes in C. Cambridge University Press, Oxford.

[15] Murase, K., Oonoki, J., Takata, H., Song, R., Angraini, A., Ausanai, P. and Matsushita, T. (2011) Simulation and Experimental Studies on Magnetic Hyperthermia with Use of Superparamagnetic Iron Oxide Nanoparticles. Radiological Physics and Technology, 4, 194202. http://dx.doi.org/10.1007/s12194-011-0123-4

[16] Kallumadil, M., Tada, M., Nakagawa, T., Abe, M., Southern, P. and Pankhurst, Q.A. (2009) Suitability of Commercial Colloids for Magnetic Hyperthermia. Journal of Magnetism and Magnetic Materials, 321, 1509-1513. http://dx.doi.org/10.1016/j.jmmm.2009.02.075

[17] Martsenyuk, M.A., Raikher, Y.L. and Shliomis, M.I. (1974) On the Kinetics of Magnetization of Ferromagnetic Particle Suspension. Soviet Physics JETP, 38, 413-416. 
Submit or recommend next manuscript to SCIRP and we will provide best service for you:

Accepting pre-submission inquiries through Email, Facebook, LinkedIn, Twitter, etc. A wide selection of journals (inclusive of 9 subjects, more than 200 journals)

Providing 24-hour high-quality service

User-friendly online submission system

Fair and swift peer-review system

Efficient typesetting and proofreading procedure

Display of the result of downloads and visits, as well as the number of cited articles

Maximum dissemination of your research work

Submit your manuscript at: http://papersubmission.scirp.org/

Or contact ojapps@scirp.org 